factors including self stigma, lack of Peer interaction, and less awareness about services in the DIC.

Method Community consultations were done initially to know the barriers to accessing services in the DIC. As physical appearance goes a long way in determining how an individual is perceived by oneself and others around, introducing sessions on beauty and healthcare by a trained beautician, was felt suitable in attracting sex workers to the Drop in Centre. A beautician was hired and oriented towards the program. The primary focus of the sessions was to showcase and teach the sex workers basic skills in dressing well and looking good using quick and cost-effective ways. Apart from this the beautician also provided few beauty services which was accessed by the sex workers. These services were charged nominally so that the amount was used to replenish the cosmetics and makeup materials. Other crucial aspects of health, such as cleanliness, hygiene and prevention of STI, female condom demonstration and distribution were covered during counselling session and one to one interactions. This innovative approach aimed towards increasing uptake and access of services proved cost effective and was received well by the most vulnerable, young sex workers, attracting them to the DIC.

Results As a result of introducing these sessions in the DIC 25 young sex workers were accessing services from the drop in centre every week. There was a steady increase in testing for STI in the introductory months itself. A peer network among the young sex workers was initiated and this helped the project to ensure that access to STI services were regular by this community. This innovative approach acted as a catalyst to improve the self-esteem of sex workers and ensured access to services there by reducing their vulnerability.

Conclusion It is imperative for prevention programs to evolve and find newer and innovative methods to address the changing needs of vulnerable communities like the female sex workers. This strategy has proved to attract young sex workers there by effectively providing them STI and other services within the program.

\section{P2-S2.23 MEASURING THE IMPACT OF HIV PREVENTION PROGRAMIME ON HIV/STI PREVALENCE AND CONDOM USE IN KARNATAKA: A CASE-CONTROL APPROACH}

doi:10.1136/sextrans-2011-050108.319

R Prakash, S Isac, B M Ramesh, P Banandur, S Moses, R Washington, P Bhattacharjee. Karnataka Health Promotion Trust, Bangalore, India

Background The important goal of Avahan programme is to reduce HIV transmission among female sex workers (FSWs) and MSM-T by increasing consistent condom use, reducing risk behaviour and timely treatment of sexually transmitted infections. In order to assess the impact of programme exposure on these outcomes, many of the earlier studies have done impact analyses using periodical Integrated Biological and Behavioural Surveys (IBBAs). Since these studies were cross-sectional in nature, they could not give true impact of programme exposure in the absence of a suitable control group. Therefore, the fact that results may have not occurred independently of the intervention, instead reflecting natural trends in HIV/STI prevalence cannot be ruled out.

Methods Using first two rounds of IBBAs conducted during 2005-2006 and 2008-2009 respectively in four districts of Karnataka namely Belgaum, Bellary, Shimoga, and Bangalore, we use Propensity Score Matching (PSM) method to demonstrate the true effect of different components of programme exposure on HIV/STI prevalence and consistent condom use with all partners. By creating a proxy group of controlled individuals (those individuals who are not exposed to programme), PSM method compares the outcome measures with each respective cases who were exposed to programme. Further, Principal Component Analysis (PCA) is used to generate a composite index by combining a set of programme exposure to analyse whether greater access to programme results into to better outcome.

Results Findings suggest that consistent condom use was higher with occasional clients in comparison to the regular clients cutting across all the districts. The consistent condom use was highest with occasional clients and regular partner in Belgaum (about 92 and 78 percent respectively) while lowest in Shimoga (55\% and $48 \%$ respectively). The extent of condom use increased for both regular and occasional partners from first survey to second one. Result shows that about $12 \%$ FSWs in Belgaum reported any STI symptoms whereas it was about more than $9 \%$ in rest of the districts. Findings further revealed the fact that greater the access to program, better was the outcome indicators. The application of PSM method clearly suggested the fact that among all the programme indicators contact of the peers with sex workers has significant positive impact on consistent condom use and HIV/STI reduction. Though the prevalence of HIV/STI was less among those sex workers who visited to clinic compared to the other groups, it was not statistically significant.

Conclusions PSM is a better alternative method to analyse the impact of programme response in the absence of a real control group. Findings clearly conclude that Peer-led Outreach "strategy is successful in scaling up HIV prevention programme, nevertheless, focus of peers on motivating key population to clinic may also contribute to HIV/STI reduction and greater condom use."

\section{Social and behavioural aspects of prevention poster session 3: General Population

P2-S3.01 STI RISK AMONG PERSONS ATTENDING BURNING MAN
IN 2010

doi:10.1136/sextrans-2011-050108.320

J L Marcus, K T Bernstein, S S Philip. San Francisco Department of Public Health, San Francisco, USA

Background Burning Man is a week-long event held annually over Labour Day in the Nevada desert, with over 50000 attendees in 2010. We aimed to assess the potential for STI transmission among San Francisco attendees and the availability and acceptability of safer-sex services at Burning Man.

Methods We conducted a survey among a convenience sample of persons who attended Burning Man in 2010. An online questionnaire on demographics, sexual practices, and safer-sex services at Burning Man was created on SurveyMonkey and advertised on two email lists, one for all attendees and one for those living in San Francisco. Attendees were invited to participate anonymously from 1 week through 6 weeks after the event. We conducted a descriptive analysis of the responses.

Results Of the 1477 persons who completed the survey during 10 September-22 October 2010, most were heterosexual men (45.1\%), heterosexual women $(30.0 \%)$, or men who have sex with men $(12.4 \%)$; of white race/ethnicity (86.5\%); and from 25 to 44 years of age $(62.5 \%)$. The majority $(70.6 \%)$ had attended the event in prior years, and $17.8 \%$ resided in San Francisco. Over one quarter $(28.5 \%)$ had been previously diagnosed with an STI. Of the $69.8 \%$ who reported having oral, vaginal, or anal sex at Burning Man, almost half $(43.5 \%)$ reported sex with new partners. Although most attendees $(86.3 \%)$ noticed that condoms were available at the event, nearly one quarter $(23.0 \%)$ of respondents with new partners had intercourse without a condom, of whom two thirds (64.9\%) did not plan to test for STIs during the month after the event. Of those planning to test, $49.3 \%$ planned to test with a private doctor, $23.9 \%$ at a community clinic, and $19.7 \%$ at an STI clinic. Only $2.6 \%$ of attendees reported that STI testing was available at the event; $41.3 \%$ said they would use the service if it were available. 